\title{
Role of reactive oxygen species-dependent protein aggregation in metabolic stress-induced necrosis
}

\author{
CHO HEE KIM ${ }^{1}$, HYUN MIN JEON ${ }^{1}$, SU YEON LEE ${ }^{1}$, EUI KYONG JEONG ${ }^{1}$, MIN KYUNG JU ${ }^{1}$, \\ BUM JOON PARK ${ }^{1}$, HYE GYEONG PARK ${ }^{2}$, SUNG-CHUL LIM ${ }^{3,4}$, SONG IY HAN ${ }^{4}$ and HO SUNG KANG ${ }^{1}$ \\ ${ }^{1}$ Department of Molecular Biology, College of Natural Sciences, ${ }^{2}$ Nanobiotechnology Center, Pusan National \\ University, Pusan 609-735; ${ }^{3}$ Research Center for Resistant Cells and ${ }^{4}$ Department of Pathology, \\ College of Medicine, Chosun University, Gwangju 501-759, Korea
}

Received February 8, 2010; Accepted April 12, 2010

DOI: 10.3892/ijo_00000657

\begin{abstract}
Cancer cells in the inner region of avascularized solid tumours experience metabolical stress by hypoxic and glucose depletion (OGD) and are prone to die by necrosis to form a necrotic core, a common feature of solid tumours. Unlike in apoptosis, where the cellular contents remain packed in the apoptotic bodies that are removed by marcrophages, necrosis is characterized by cell membrane rupture, and the release of many cellular proteins including tumour promoting cytokine high mobility group box 1 (HMGB1) into the extra-cellular space. Although ROS produced by metabolic stress are known to cause membrane damage leading to the plasma membrane rupture, its molecular mechanism remains unclear. In this study, we show that some cellular proteins including pro-apoptotic molecules p53, caspase-3, and caspase- 9 and a pro-autophagic molecule beclin 1 are not released into the extracellular space but rather aggregated in the cytosol during GD-induced necrosis and that the protein aggregation occurs in a ROS-dependent manner. We also found that Snail, the transcription factor that is induced by GD, was not translocated to the nucleus and aggregated in the cytosol. In addition, Snail interference appeared to block metabolic stress-induced protein aggregation, indicating a critical role(s) of Snail in the protein aggregation. These results demonstrate that in metabolically stressed cancer cells, ROS induce a specific set of cellular proteins to form insoluble aggregates that are highly toxic to cells and trigger the necrosis-associated membrane rupture and HMGB1 release to promote tumour progression.
\end{abstract}

\section{Introduction}

When cancer cells grow and form solid tumours, their center becomes distant from surrounding blood vessels and

Correspondence to: Dr Ho Sung Kang, Department of Molecular Biology, College of Natural Sciences, Pusan National University, Pusan 609-735, Korea

E-mail: hspkang@pusan.ac.kr

Key words: necrosis, metabolic stress, ROS, protein aggregate, Snail experience hypoxic and glucose depletion (OGD) stress (1-3). Under circumstances of metabolic or hypoxic stresses, tumour cells either adapt to survive against the potentially lethal effects of metabolic constraint or undergo cell death. In solid tumours, metabolic stress-induced cell death mostly occurs by necrosis because tumour cells become defective in apoptotic and/or autophagic program during carcinogenesis $(1,3)$. In fact, necrotic core is commonly found in the inner region of poorly vascularized tumours and is associated with poor prognosis. $(1,3,4)$. Unlike in apoptosis, where the cellular contents remain packed in the apoptotic bodies that are removed by phagocytes or neighboring cells, thereby resulting in cellular deletion without inflammation (5), during necrosis the cell membrane is ruptured, and many cellular proteins including the nuclear protein high mobility group box 1 (HMGB1) and the S100 family of molecules are relased into the extra-cellular space, thus provoking the inflammatory response and facilitating tumour progression (6-8). Previously, we also showed that CuZnSOD, but not the antioxidants MnSOD and catalase, is released into the extracellular space in an active form upon glucose depletion (GD), one of metabolic stresses, thereby accelerating ROS damage and facilitating necrotic cell death $(9,10)$. Unlike tumour suppressive apoptosis (11-13) or autophagic cell death (14-15), necrosis has been implicated in tumour growth and aggressiveness (16-20). ROS, $\mathrm{Ca}^{2+}$, and other factors have been demonstrated to be involved in necrosis that occurs in response to metabolic stress, DNA damage and TNF- $\alpha$ $(21,22)$. Although ROS is known to cause membrane damage leading to plasma membrane rupture, its exact mechanism remains unclear.

Previously, we have shown that metabolic stress induces necrosis and HMGB1 release through mitochondrial ROS production in several cancer cells (10). Here we show that a specific set of cellular proteins including pro-apoptotic molecules p53, caspase-3, and caspase-9 and a pro-autophagic molecule beclin 1 are aggregated in a ROS-dependent manner during GD-induced necrotic cell death. We also found that Snail, the transcription factor that is induced by GD, was not translocated to the nucleus and aggregated in the cytosol. In addition, Snail interference appeared to block metabolic stressinduced protein aggregation, indicating a critical role(s) of 
Snail in the protein aggregation. These results suggest that ROS induced by metabolic stress induces the formation of protein aggregates that are highly toxic to cells and could trigger the necrosis-associated membrane rupture and HMGB1 release to promote tumour progression.

\section{Materials and methods}

Cell culture and chemical treatment. A549, MDA-MB231, and MCF-7 cells were obtained from American Type Culture Collection and maintained in RPMI-1640 or DMEM containing $10 \%$ FBS and treated with GD or chemicals as described previously (10).

Western blotting, HMGB1 release assay, and protein aggregation assay. Western blotting with antibodies to Snail [polyclonal anti-Snail antiserum prepared as previously described (23), beclin 1, p53, caspase-3, caspase-9, (Santa Cruz, CA), ERK1/2 (Cell Signaling, MA), $\alpha$-tubulin (Biogenex, CA), B-tubulin (Sigma), or HMGB1 (BD Pharmingen, CA) and HMGB1 release assay were performed as described previously (10)]. RIPA soluble fraction was obtained by lysing cells in RIPA buffer $(50 \mathrm{mM}$ Tris $\mathrm{pH} 7.5,200 \mathrm{mM} \mathrm{NaCl}, 1 \%$ Triton X-100, $0.5 \%$ deoxycholate, $0.1 \%$ sodium dodecyl sulfate, $1 \mathrm{mM}$ EDTA) for $20 \mathrm{~min}$ on ice, followed by centrifugation at $14,000 \mathrm{rpm}$ for $15 \mathrm{~min}$ and the RIPA insoluble fraction was suspended in PBS and boiled in 2X Laemmli sample buffer. Cells were also fractionated into cytosol (F1), membranes (F2), nuclei (F3), and cytoskeleton and insoluble fraction (F4) using a Subcellular Proteo Extract ${ }^{\circledR}$ kit (Calbiochem, VWR International Ltd., Lutterworth). The purity of each fraction was analyzed by immunoblotting using antibodies against cytosolic CuZnSOD and procaspase-3 (F1), mitochondrial MnSOD, peroxixomal catalase (F2), nuclear HMGB1 (F3).

Short hairpin RNA interference. pSUPER-Snail-shRNA was generated from annealed oligonucleotide (5'-GATCCCCGC GAGCTGCAGGACTCTAATTCAAGAGATTAGAGTCC $\underline{\text { TGCAGCTCGCTTTTTA-3' and 5'-AGCTTAAAAAGCGA }}$ GCTGCAGGACTCTAATCTCTTGAATTAGAGTCCT GCAGCTCGCGGG-3' (Accession No. NM_005985) (24) and control shRNA was generated from annealed oligonucleotides (5'-GATCCCCAATTCTCCGAACGTGTCACGTT TCAAGAGAACGTGACACGTTCGGAGAATTTTTTTA-3' and 5'-AGCTTAAAAAAATTCTCCGAACGTGTCACGTT CTCTTGAAACGTGACACGTTCGGAGAATTGGG-3') (23) inserted into the HindIII and BglII sites of pSUPER.gfp/neo (Oligoengine Platform, Seattle, WA). All target sequences were designed and verified as specific for Snail by Blast search against the human genome and reverse transcription-PCR, respectively. pSUPER-shCon and pSUPER-shSnail vectors were transfected using jetpei and selection performed with $1 \mathrm{mg} / \mathrm{ml} \mathrm{G} 418$. Several clones were isolated after shRNA transfection in each cell type and individually characterized.

Hoechst 33342 (HO)/propidium iodide (PI) staining, immunofluorescence, and confocal microscopy. HO/PI staining was performed as described previously (10). For immunofluorescence and confocal microscopy, cells were fixed with $100 \%$ methanol for $10 \mathrm{~min}$ at $-20^{\circ} \mathrm{C}$ and then incubated with blocking buffer (PBS containing $0.1 \%$ Triton X-100 and anti-goat antibody) for $1 \mathrm{~h}$ at RT. After washing with ice-cold PBS, the cells were incubated with anti-Snail (Abcam, Cambridge, UK), anti-p53 and anti-capase 9 antibodies (Santa Cruz) diluted in blocking buffer and then reacted with a FITC- or rhodamineconjugated secondary antibody (Santa Cruz). After mounting, the cellular localization of Snail, p53, and caspase-9 was observed by fluorescence microscopy or laser scanning confocal microscopy (LSM510, Carl Zeiss). Intracellular $\mathrm{H}_{2} \mathrm{O}_{2}, \mathrm{O}_{2}{ }^{-}$and mitochondrial ROS were detected using the 2', 7'-dichlorofluorescin diacetate (DCFH-DA (Molecular Probes, $50 \mu \mathrm{M}$ ), dihydroethidium (HE, Molecular Probes, $10 \mu \mathrm{M}$ ), or MitoTracker Red CM- $\mathrm{H}_{2}$ XRos (Molecular Probe, $50 \mathrm{nM}$ ) by fluorescence microscopy.

Transmission electron microscopy. For TEM, collected cells were fixed in $2.5 \%$ glutaraldehyde with $0.1 \mathrm{M}$ cacodylate buffer ( $\mathrm{pH} 7.2$ ) for $2 \mathrm{~h}$ at $4^{\circ} \mathrm{C}$ and washed twice with cold $\mathrm{PBS}$, postfixed in $\mathrm{OsO}_{4}$, dehydrated in graded ethanol, and embedded in epon mixture. Sections were prepared with ultramicrotome (MT-7000), mounted in copper grids, and counterstained with uranyl acetate and lead citrate. Photographs were taken using electron microscope (Hitachi H-7600).

\section{Results and Discussion}

ROS-dependent protein aggregation in metabolic stressinduced necrosis. Previously, we have shown that GD, one of stresses that cause metabolic stress in tumours $(1,3)$, induced necrosis in A549 cells as determined by Hoechst 33342 (HO)/propidium iodide staining (10). DNA-binding dyes HO crosses the plasma membrane of all cells, whether they are damaged or not, causing a blue fluorescence of their nuclei, but PI only penetrates cells with damaged membranes and leads to nuclear fluorescence. Thus, intact blue nuclei, condensed/fragmented blue nuclei, condensed/fragmented pink nuclei, and intact pink nuclei were considered viable, early apoptotic, late apoptotic (secondary necrotic), and necrotic cells, respectively. GD increased the population of the cells that had intact pink nuclei (HO/PI staining). In A549 cells, pretreatment of NAC (the ROS-quenching agent $\mathrm{N}$ acetyl-L-cysteine) repressed GD-induced increase in population of PI-positive cells (Fig. 1A) and HMGB1 release (10) and increased cells with condensed/fragmented blue nuclei (HO/PI double staining), thus indicating that NAC switches GD-induced necrotic cell death to apoptosis. TEM also showed that NAC prevented GD-induced necrosis and switched the cell death mode to apoptosis in A549 cells (Fig. 1B). In case of HepG2 and MDA-MB231 cells, NAC also prevented necrosis but without increasing apoptotic cells (Fig. 1A). When GD-induced necrosis was inhibited by NAC in these cells, apoptosis did not occur as an alternative death mechanism. Instead, autophagic vacuole-like structures with contents undergoing digestion were observed (Fig. 1B). Thus, ROS play a critical role(s) in metabolic stress-induced cell death mode determination in cancer cells.

In the course of this study, we found that GD increased the amount of RIPA-insoluble proteins (Fig. 1C). Especially, pro-apoptotic molecules p53, pro-caspase 3 , and pro-caspase 9 

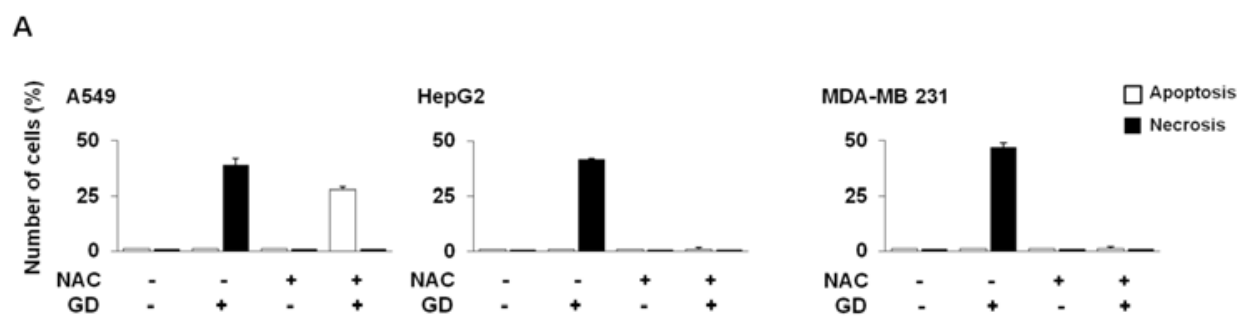

B

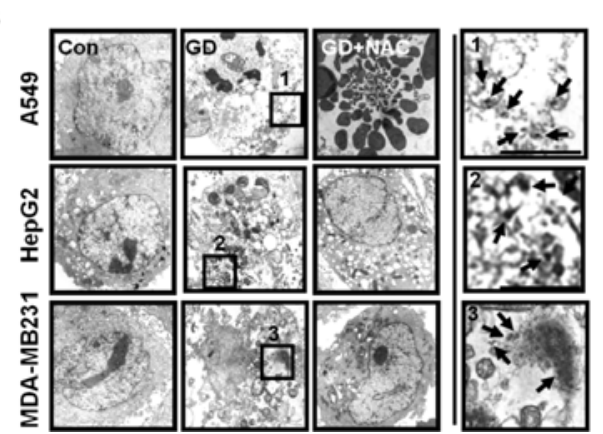

$\mathrm{C}$

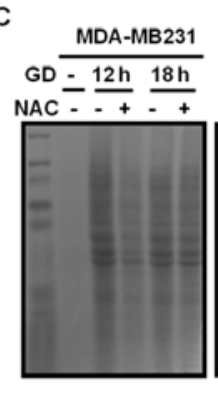

D

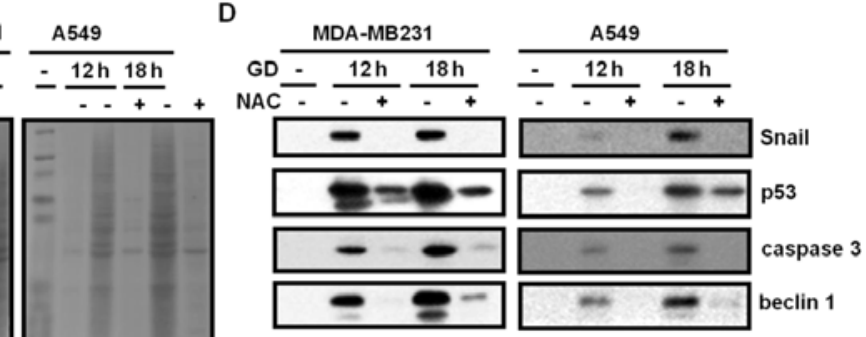

Figure 1. (A) A549, HepG2 and MDA-MB231 cells pretreated with NAC were exposed to GD for 18 h and then stained with HO/PI and observed by fluorescence microscopy and necrotic and apoptotic cells were scored. Results (500-800 cells in each group) are expressed as the means \pm SEM from three independent experiments. (B) A549, HepG2 and MDA-MB231 cells pretreated with NAC were exposed to GD for 18 h and observed by transmission electron microscopy. Arrow, electron-dense aggregates. (C and D) MDA-MB231 and A549 cells were cultured in normal growth medium or GD medium in the presence or absence of $10 \mathrm{mM}$ NAC for 12 and $18 \mathrm{~h}$. RIPA-soluble and -insoluble fractions were obtained and analyzed by SDS-PAGE/Ponceau S staining (C) and Western blotting with antibodies to Snail, p53, pro-caspase 3, and beclin 1 (D).

and a pro-autophagic molecule beclin 1 were found in RIPAinsoluble fractions (Fig. 1D). GD-induced insolublization of p53 and pro-caspase 9 was confirmed by immunofluorescence microscopy (see Figs. 3D and 4B). Upon GD, p53 and procaspase 9 yielded a cytoplasmic distribution in an aggregated form and the aggregates first appeared as small dots and progressively increased in size over time and formed a patchy pattern surrounding the nuclei. Additionally, TEM showed massive accumulations of closely packed electrondense particles in the cytosol, particularly in the perinuclear region after GD treatment (Fig. 1B).

Oxidative stress is known to trigger the formation of oxidatively modified proteins that tend to aggregate, thereby causing cell death. NAC was shown to block GD-induced aggregate formation (Fig. 1C and D). TEM also showed that NAC blocked GD-induced aggregate formation (Fig. 1B). These results indicate that metabolic stress-induced protein aggregation occurs ROS-dependently.

p53 is inactivated in metabolic stress-induced protein aggregates. To verify whether the proteins in aggregates are still active, we pretreated A549 cells with etoposide that is known to induce p21/Waf1 expression and apoptosis in a p53-dependent manner, and treated them with GD. GD treatment abolished etoposide-induced p21/Waf1 expression (Fig. 2A) and apop-tosis (Fig. 2B), indicating that p53 is severely damaged and inactivated during necrosis.

Snail is induced by metabolic stress and aggregated in the cytosol. Recently, we found that Snail, the transcription factor that induces the epithelial-mesenchymal transition (EMT) through repressing E-cadherin expression, is induced by GD and plays a critical role in metabolic stress-induced necrosis (unpublished data). In this study, we analyzed the

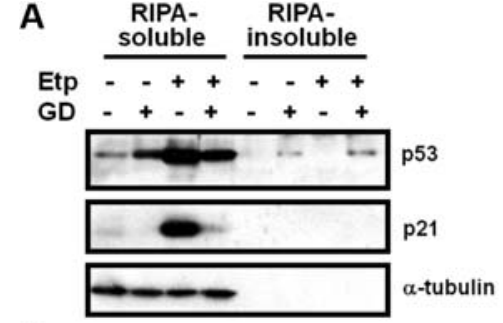

B

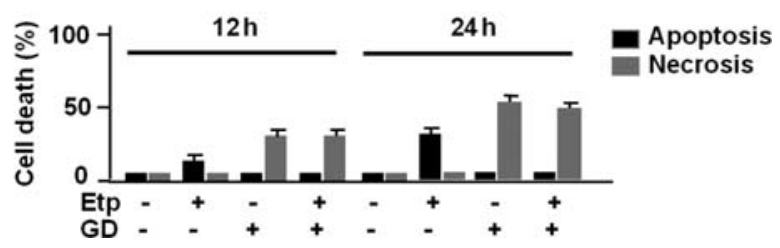

Figure 2. GD treatment abolished etoposide-induced p21 expression (A) and apoptosis in A549 cells (B). GD treatment abolished etoposide-induced apoptosis in A549 cells.

subcellular distribution of Snail in metabolically stressed cells by confocal microscopy. We observed the metabolic stressed-induced Snail was detected exclusively in cytosol as large amorphous protein aggregates that were randomly dispersed throughout the cytoplasmic compartment, with no detectable protein in the nucleus (Fig. 3A). To further characterize the status of Snail, we performed partition experiments using RIPA fractionation. Although Snail was found predominantly in the RIPA-soluble fractions, a signifianct amount of Snail was also detectable in the RIPA-insoluble fractions at $12 \mathrm{~h}$ of GD, indicating that Snail forms insoluble aggregates upon GD (Fig. 3B). To confirm the distribution of Snail in the 

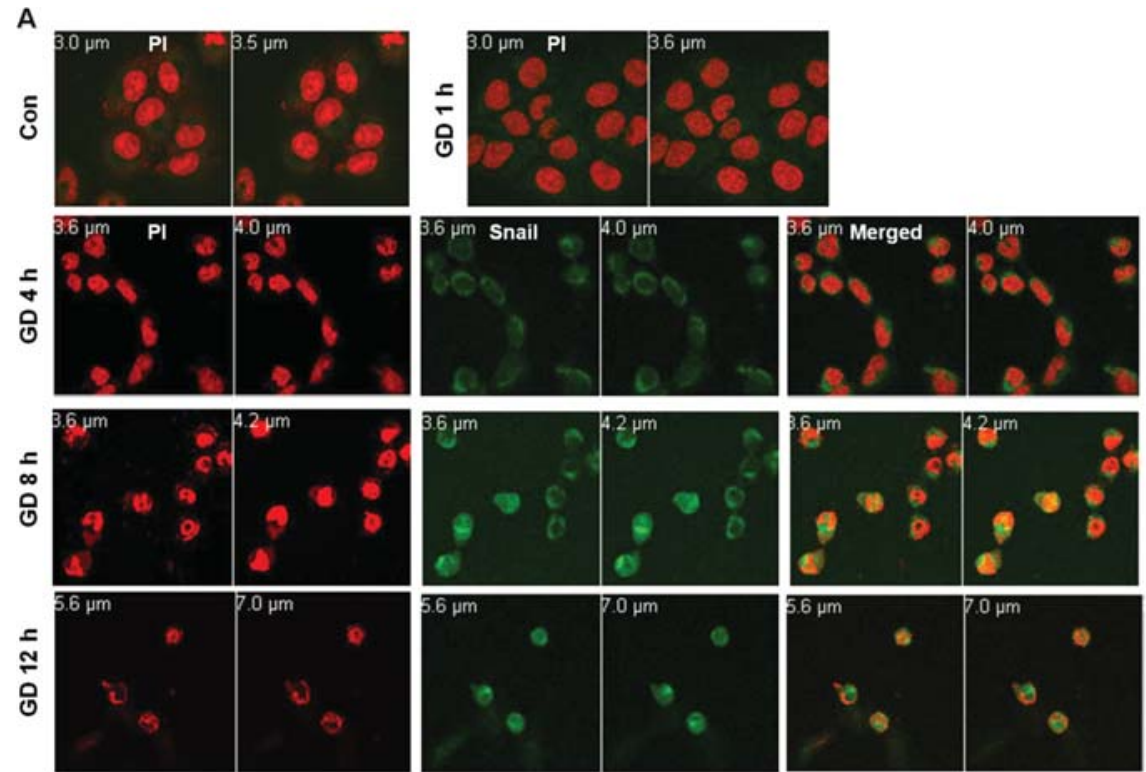

B

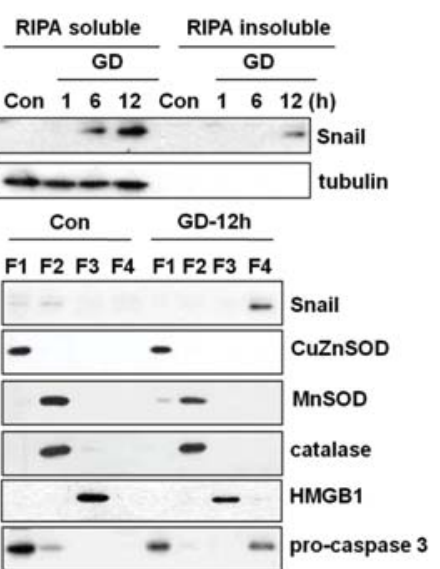

E
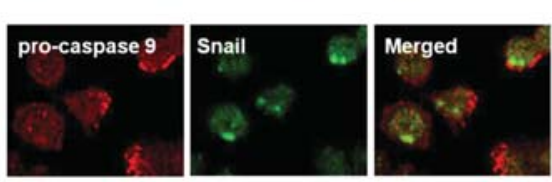

F

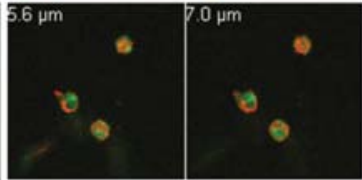

D

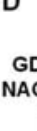

$\frac{\text { A549 }}{-++} \frac{\text { HepG2 }}{-++} \frac{\text { MDA-MB231 }}{-++t}$

NAC - - + + - - + + + +
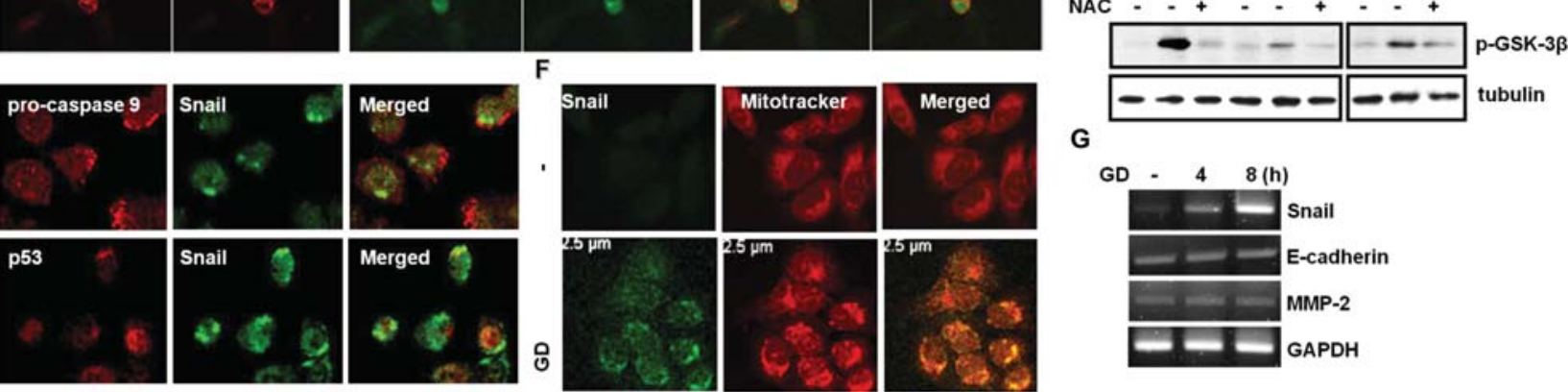

G
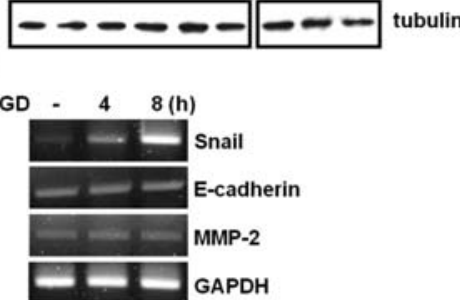

Figure 3. Metabolic stress-induced Snail localizes as insoluble aggregates in cytosol. (A) A549 cells were treated with GD medium for 0 to $12 \mathrm{~h}$ and stained with anti-Snail antibody and PI and analyzed by confocal laser scanning microscopy. (B) MDA-MB231 cells were cultured in normal growth medium or GD medium for $12 \mathrm{~h}$. RIPA-soluble and -insoluble fractions were obtained and analyzed by SDS-PAGE and Western blotting with antibodies to Snail and tubulin. (C) MDA-MB231 cells were cultured in normal growth medium or GD medium for $12 \mathrm{~h}$ and fractionated into cytosol (F1), membrane and organelles (F2), nuclei (F3), and cytoskeleton and insoluble fraction (F4) using a Subcellular ProteoExtract ${ }^{\circledR}$ kit. The purity of each fraction was analyzed by immunoblotting using antibodies against cytosolic CuZnSOD and pro-caspase 3 (F1), mitochondrial MnSOD, peroxisomal catalase (F2), and nuclear HMGB1 (F3). (D) MDA-MB231 cells were treated with GD medium for $12 \mathrm{~h}$ and stained with antibodies to Snail, p53 and pro-caspase 9 and then observed by confocal laser scanning microscopy. (E) A549 cells were treated with GD medium for $12 \mathrm{~h}$ and incubated with Mitotracker Red CMX-Ros and stained with anti-Snail antibody and analyzed by confocal laser scanning microscopy. (F) A549, HepG2, and MDA-MB231 cells were pretreated with NAC for $1 \mathrm{~h}$ and then treated with GD medium for $12 \mathrm{~h}$ and analyzed by Western blotting with antibodies to phospho-GSK-3ß and $\alpha$-tubulin. (G) A549 cells were treated with GD medium for $12 \mathrm{~h}$ and analyzed by RT-PCR using primers for Snail, E-caderin, MMP-2, and GAPDH.

A

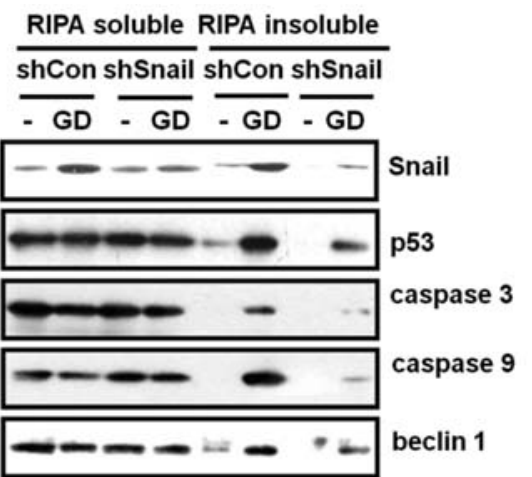

B

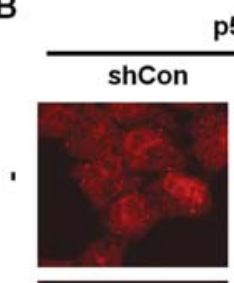

p53

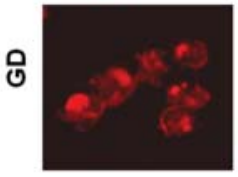

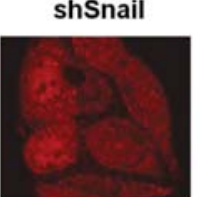

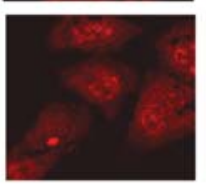

pro-caspase 9

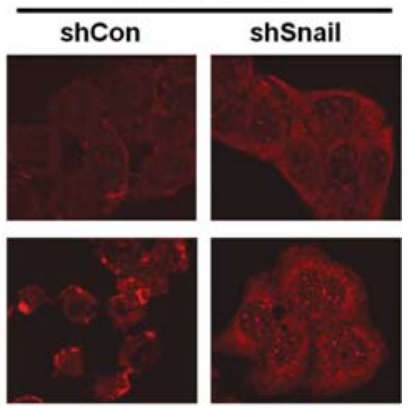

Figure 4. (A) MDA-MB231 cells stably transfected with shCon and shSnail cells were cultured in normal growth medium or GD medium for 12 h and RIPAsoluble and -insoluble fractions were obtained and analyzed by SDS-PAGE and Western blotting with antibodies to Snail, p53, pro-caspase 3, pro-caspase 9, and beclin 1. (B) MDA-MB231 cells stably transfected with shCon and shSnail were treated with GD medium for $12 \mathrm{~h}$. The cells were stained with antibodies to 53 and caspase-9 and then observed by confocal laser scanning microscopy.

aggregates, we carried out subcellular fractionation of cells. Cells were fractionated into cytosol (F1), membranes and organelles (F2), nuclei (F3), and cytoskeleton and insoluble fraction (F4) using a Subcellular ProteoExtract ${ }^{\circledR}$ kit (Calbiochem, VWR International Ltd., Lutterworth). Subcellular fractionation also revealed GD-induced insoluble aggregate 
formation of Snail (Fig. 3C). Snail was found mainly in the cytoskeleton and insoluble fraction (F4). It was deduced, therefore, that Snail is essentially aggregated in the cytosol.

Then, we investigated whether the cytoplasmic Snail aggregate is colocalized with p53 and pro-caspase 9. As shown in Fig. 3D, the subcellular distribution of Snail was quite different from those of p53 and caspase, although a partial colocalization of Snail with p53/pro-caspase 9 in aggregate structures was detectable upon prolonged GD treatment. This result suggests that there is no physical interaction between Snail and p53, and pro-caspase 3 aggregate. We also observed that Snail colocalized with mitochondria that were revealed by Mitotracker-Red. Mitochondrial localization of Snail was defined by yellow spots, indicating overlap of FITC (Snail) and Mitotracker-Red (Fig. 3E).

Snail is known to localize predominantly to the nucleus of most cells, where it represses the transcription of downstream genes through a sequence-specific E-box element. However, in this study, Snail was not detected in the nucleus, but instead detected in cytosol as RIPA-insoluble aggregates. Snail is known to be targeted for GSK-3ß-mediated nuclear export and proteasomal degradation. GSK-3ß appeared to be phosphorylated at Ser-9 and inactivated upon GD (Fig. 3F). In addition, GD-induced phosphorylation of GSK-3ß was suppressed by NAC treatment, indicating ROS-dependent GSK-3ß inactivation (Fig. 3F). The GSK-3ß inhibition may be closely linked to an increase in the stability and aggregation of Snail protein in the cytosol.

Snail represses E-cadherin expression to induce the epithelial-mesenchymal transition (EMT) and confers epithelial cells with migratory and invasive properties during both embryonic development and tumour progression (25-29). However, GD-induced Snail could not affect mRNA levels of E-cadherin and another target of Snail, matrix metalloproteinase (MMP)-2 (Fig. 3G) nor induced EMT (not shown). These results demonstrate that the aggregated Snail may be functionally impaired in its transcriptional activity to regulate gene expression.

Snail plays a critical role(s) in protein aggregation during metabolic stress-induced necrosis. Since Snail plays a critical role in metabolic stress-induced necrosis, we examined whether Snail affects metabolic stress-induced protein aggregation. We found that Snail shRNA significantly blocked the aggregation of p53, caspases, and beclin 1 in response to GD as revealed by RIPA fractionation (Fig. 4A), and by immunofluorescence microscopy (Fig. 4B). These results suggest that Snail plays a critical role(s) in metabolic stress-induced protein aggregation.

In normal conditions, Snail down-regulates E-cadherin to induce EMT; however, under circumstances of highly oxidative stress, it is aggregated and accumulated in the cytosol and may lose its transcriptional activity to down-regulate E-cadherin and to induce EMT. Cellular proteins clumped into insoluble aggregates by a variety of stimuli including oxidative stress are mostly removed by autophagy or the ubiquitin-proteasome pathways (30-32). Thus, Snail may suppress ubiquitin-proteasome pathways and/or in autophagy that are responsible for degradation of insoluble aggregates (Fig. 4B). The possible involvement of Snail in autophagy or the ubiquitin-proteasome pathways is under investigation.
Biological relevance for ROS-dependent protein aggregation in necrosis. Necrosis is characterized by the cell membrane rupture, and the release of intracellular factors including HMGB1 and the S100 family into the extracellular space (6-8). CuZnSOD, but not the other antioxidants MnSOD and catalase, was also shown to be released into the extracellular space $(9,10)$. However, we observed that although intracellular levels of proteins including $\alpha$-actin, $\alpha$-tubulin, $\beta$ tubulin, p53, caspase- 3 , and caspase- 9 were decreased during necrosis, they were not detected in the culture medium (9). Here we show that these proteins are aggregated in the cytosol, in contrast to general concepts which state that necrotic cell death causes the release of most cellular proteins due to cell membrane rupture. The molecular mechanism for selective protein aggregation and release remains to be elucidated. Such a difference may be due to their differential sensitivity to oxidative stress; while some proteins may be highly sensitive to ROS and easily form insoluble aggregates, others are rather resistant and do not form aggregates. Insoluble protein aggregates are known to be toxic to cells and cause cell death especially necrosis (30-32). Abnormal protein aggregation has been found in focal ischemic region of brain that leads to neuronal death and heart ischemia as well as in many neurodegenerative diseases, including Alzheimer's disease, Parkinson's disease, and Huntington's disease (30-34). Among protein aggregates such as oligomeric globular assemblies, protofibrils, and ring-like structures, ring-like structures could form non-specific membrane pores that lead to necrosis. Thus, proteins that are highly sensitive to ROS could form aggregates that trigger the necrosis-associated membrane rupture and release of other cellular proteins including HMGB1 in the extracellular space to promote tumour progression. Studies on necrosis-associated protein aggregation have important clinical implications and could be informative in the development of more effective therapeutic approaches for tumours.

\section{Acknowlegdements}

This study was supported by the National Research Foundation of Korea (NRF) grant funded by the Korea government (MEST) (2009-0059210), and a grant of the Korea Healthcare technology R\&D Project, Ministry for Health, Welfare, Republic of Korea (A080303).

\section{References}

1. Gatenby RA and Gillies RJ: Why do cancers have high aerobic glycolysis? Nat Rev Cancer 4: 891-899, 2004.

2. Harris AL: Hypoxia - a key regulatory factor in tumour growth. Nat Rev Cancer 2: 38-47, 2002.

3. Tomes L, Emberley E, Niu Y, Troup S, Pastorek J, Strange K, Harris A and Watson PH: Necrosis and hypoxia in invasive breast carcinoma. Breast Cancer Res Treat 81: 61-69, 2003.

4. Cheville JC, Lohse CM, Zincke H, Weaver AL and Blute ML: Comparisons of outcome and prognostic features among histologic subtypes of renal cell carcinoma. Am J Surg Pathol 27: 612-624, 2003.

5. Kanduc D, Mittelman A, Serpico R, Sinigaglia E, Sinha AA, Natale C, Santacroce R, Di Corcia MG, Lucchese A, Dini L, Pani P, Santacroce S, Simone S, Bucci R and Farber E: Cell death: apoptosis versus necrosis (review). Int J Oncol 21: 165-170, 2002. 
6. Lotze MT and Tracey KJ: High-mobility group box 1 protein (HMGB1): nuclear weapon in the immune arsenal. Nat Rev Immunol 5: 331-342, 2005.

7. Scaffidi P, Misteli T and Bianchi ME: Release of chromatin protein HMGB1 by necrotic cells triggers inflammation. Nature 418: 191-195, 2002

8. Zeh HJ III and Lotze MT: Addicted to death: invasive cancer and the immune response to unscheduled cell death. J Immunother 28: 1-9, 2005.

9. Han SI, Duong HQ, Choi JE, Lee TB, Kim CH, Lee SY, Jeon HM, Shin SH, Lim SC and Kang HS: Hyperthermia switches glucose depletion-induced necrosis to apoptosis in A549 lung adenocarcinoma cells. Int J Oncol 32: 851-860, 2008.

10. Kim CH, Han SI, Lee SY, Youk HS, Moon JY, Duong HQ, Park MJ, Joo YM, Park HG, Kim YJ, Yoo MA, Lim SC and Kang HS: Protein kinase C-ERK1/2 signal pathway switches glucose depletion-induced necrosis to apoptosis by regulating superoxide dismutases and suppressing reactive oxygen species production in A549 lung cancer cells. J Cell Physiol 211: 371-385, 2007.

11. Danial NN and Korsmeyer SJ: Cell death: critical control points. Cell 116: 205-219, 2004.

12. Hengartner MO: The biochemistry of apoptosis. Nature 407: 770-776, 2000

13. Slee EA, O'Connor DJ and Lu X: To die or not to die: how does p53 decide? Oncogene 23: 2809-2818, 2004.

14. Hippert MM, O'Toole PS and Thorburn A: Autophagy in cancer: good, bad, or both? Cancer Res 66: 9349-9351, 2006.

15. Liang XH, Jackson S, Seaman M, Brown K, Kempkes B, Hibshoosh $\mathrm{H}$ and Levine B: Induction of autophagy and inhibition of tumourigenesis by beclin 1. Nature 402: 672-676, 1999

16. Degenhardt K, Mathew R, Beaudoin B, Bray K, Anderson D, Chen G, Mukherjee C, Shi Y, Gelinas C, Fan Y, Nelson DA, Jin S and White E: Autophagy promotes tumour cell survival and restricts necrosis, inflammation, and tumourigenesis. Cancer Cell 10: 51-64, 2006.

17. Jin S and White E: Role of autophagy in cancer: management of metabolic stress. Autophagy 3: 28-31, 2007.

18. Schlueter C, Weber H, Meyer B, Rogalla P, Roser K, Hauke S and Bullerdiek J: Angiogenetic signaling through hypoxia: HMGB1: an angiogenetic switch molecule. Am J Pathol 166: 1259-1263, 2005.

19. Taguchi A, Blood DC, del Toro G, Canet A, Lee DC, Qu W Tanji N, Lu Y, Lalla E, Fu C, Hofmann MA, Kislinger T, Ingram M, Lu A, Tanaka H, Hori O, Ogawa S, Stern DM and Schmidt AM: Blockade of RAGE-amphoterin signalling suppresses tumour growth and metastases. Nature 405: 354-360, 2000 .
20. Vakkila $\mathbf{J}$ and Lotze MT: Inflammation and necrosis promote tumour growth. Nat Rev Immunol 4: 641-648, 2004.

21. Golstein P and Kroemer G: Cell death by necrosis: towards a molecular definition. Trends Biochem Sci 32: 37-43, 2007.

22. Zong WX and Thompson CB: Necrotic death as a cell fate. Genes Dev 20: 1-15, 2006.

23. Yook JI, Li XY, Ota I, Hu C, Kim HS, Kim NH, Cha SY, Ryu JK, Choi YJ, Kim J, Fearon ER and Weiss SJ: A Wnt-Axin2GSK3beta cascade regulates Snail1 activity in breast cancer cells. Nat Cell Biol 8: 1398-1406, 2006.

24. Kajita M, McClinic KN and Wade PA: Aberrant expression of the transcription factors snail and slug alters the response to genotoxic stress. Mol Cell Biol 24: 7559-7566, 2004.

25. Boutet A, De Frutos CA, Maxwell PH, Mayol MJ, Romero J and Nieto MA: Snail activation disrupts tissue homeostasis and induces fibrosis in the adult kidney. EMBO J 25: 5603-5613, 2006.

26. Cano A, Perez-Moreno MA, Rodrigo I, Locascio A, Blanco MJ, del Barrio MG, Portillo F and Nieto MA: The transcription factor snail controls epithelial-mesenchymal transitions by repressing E-cadherin expression. Nat Cell Biol 2: 76-83, 2000.

27. Carver EA, Jiang R, Lan Y, Oram KF and Gridley T: The mouse snail gene encodes a key regulator of the epithelialmesenchymal transition. Mol Cell Biol 21: 8184-8188, 2001.

28. Nieto MA: The snail superfamily of zinc-finger transcription factors. Nat Rev Mol Cell Biol 3: 155-166, 2002.

29. Peinado H, Olmeda D and Cano A: Snail, Zeb and bHLH factors in tumour progression: an alliance against the epithelial phenotype? Nat Rev Cancer 7: 415-428, 2007.

30. Lansbury PT and Lashuel HA: A century-old debate on protein aggregation and neurodegeneration enters the clinic. Nature 443: 774-779, 2006.

31. Ross CA and Poirier MA: Opinion: What is the role of protein aggregation in neurodegeneration? Nat Rev Mol Cell Biol 6: 891-898, 2005

32. Syntichaki $P$ and Tavernarakis $\mathrm{N}$ : The biochemistry of neuronal necrosis: rogue biology? Nat Rev Neurosci 4: 672-684, 2003.

33. Hu BR, Janelidze S, Ginsberg MD, Busto R, Perez-Pinzon M, Sick TJ, Siesjo BK and Liu CL: Protein aggregation after focal brain ischemia and reperfusion. J Cereb Blood Flow Metab 21: $865-875,2001$

34. Hu BR, Martone ME, Jones YZ and Liu CL: Protein aggregation after transient cerebral ischemia. J Neurosci 20: 31913199,2000 